\title{
Strain Induced Dewetting of a Molecular System: Bimodal Growth of PTCDA on NaCl
}

\author{
S. A. Burke, ${ }^{1}$ W. Ji, ${ }^{1,2}$ J. M. Mativetsky, ${ }^{1}$ J. M. Topple, ${ }^{1}$ S. Fostner, ${ }^{1}$ H.-J. Gao, ${ }^{2}$ H. Guo, ${ }^{1}$ and P. Grütter ${ }^{1}$ \\ ${ }^{1}$ Centre for the Physics of Materials and Department of Physics, McGill University, Montreal, Quebec, Canada H3A 2T8 \\ ${ }^{2}$ Beijing National Laboratory for Condensed Matter Physics, Institute of Physics, Chinese Academy of Sciences, \\ P.O. Box 603, Beijing 100080, China
}

(Received 23 November 2007; published 9 May 2008)

\begin{abstract}
Submonolayer coverages of the molecule 3,4,9,10-perylene tetracarboxylic dianhydride (PTCDA) deposited on $\mathrm{NaCl}(001)$ surfaces were imaged with high resolution noncontact-atomic force microscopy. Two island types were observed: monolayer islands with a $p 3 \times 3$ epitaxy at low coverage and a mixture of these and bulklike crystallites at higher coverage. The transition between the pure monolayer islands and mixed islands occurs at $\sim 0.85 \mathrm{ML}$, corresponding to a complete $p 3 \times 3$ layer. Calculations show the $p 3 \times 3$ epitaxy to be incompatible with a multilayer crystal of PTCDA. Consequently, the growth of additional layers results in an adaptation of the interface structure forcing a dewetting transition.
\end{abstract}

DOI: 10.1103/PhysRevLett.100.186104

The promise of utilizing organic molecules as active components in electronic and optoelectronic devices has lead to great interest in their characterization on solid substrates [1,2]. Structural properties of these molecular thin films and crystallites has proven to be of importance as the morphology of the film influences the electronic properties to be used $[3,4]$. As such, the growth mechanisms and resulting morphologies of organic materials on inorganic substrates, including insulators which will be needed to isolate device regions, are of considerable interest in the pursuit of constructing optimized devices.

Both the energetics of a system and kinetic factors during growth determine the morphology of the resulting structures. While a particular growth mode is usually preferred, under certain conditions there can be a transition from layer growth to 3D islands by removal of the wetting layer, often termed "dewetting." Several recent studies have reported molecular dewetting, for example: perylene on $\mathrm{Au}$ and $\mathrm{Cu}$ [5], pentacene on $\mathrm{Au}$ [6], phenylthiophene fulgide on glass [7], and $C_{60}$ on alkali halides [8]. On metallic substrates, molecular dewetting is thought to be a result of strain and a reorientation of the molecules from the typical coplanar adsorption geometry for monolayer films to a tilted or standing up phase consistent with the bulk crystal structure $[5,6]$. While there is some evidence for this molecular scale mechanism for molecules adsorbed on metal surfaces, there has been little study of such dynamical processes and their molecular scale origins on insulating surfaces [8].

The molecule 3,4,9,10-perylene tetracarboxylic dianhydride (PTCDA) has been studied extensively as a prototypical organic semiconductor [3]. On many surfaces, crystallites are formed with a structure much like the two bulk polymorphs " $\alpha$ " and " $\beta$ " [9]. These crystallites are most commonly oriented with the (102) plane parallel to the surface such that the planar PTCDA molecule lies flat on the surface and is arranged in layers of a "herringbone" lattice. Coverages exceeding $1 \mathrm{ML}$ (monolayer) were
PACS numbers: 68.35.bm, 68.37.Ps, 68.43.Hn, 68.55.am

studied on several alkali halide surfaces, including $\mathrm{NaCl}$, by Schlettwein et al. [10]. In that study, in situ optical spectroscopy showed monomerlike emission during the initial stages of growth, indicating weakly interacting species on the surface, disappearing at $\sim 1 \mathrm{ML}$ where a lower energy excitonic peak dominates, indicating the presence of bulklike crystallites [10]. The authors noted the need for molecular resolution imaging to determine the structures in order to fully understand their observations; however, such studies have not been forthcoming. More recently, PTCDA has been studied on $\mathrm{KBr}$ [11] and pit templated $\mathrm{KBr}$ $[12,13]$ with molecular resolution using noncontact-atomic force microscopy (nc-AFM) to determine the structure of crystallites, and in several cases these molecular resolution images have lead to determination of the epitaxy of molecular systems on insulators $[8,12,14]$.

We describe herein the morphology of submonolayer coverages of PTCDA on the model insulating substrate $\mathrm{NaCl}$. The use of nc-AFM allows molecular resolution imaging and determination of the detailed structure of the crystallites and monolayer islands observed. Based on these structures and accompanying calculations the molecular scale origin of a dewetting transition is elucidated. This bimodal growth with a dewetting transition triggered by increasing coverage is similar to that observed for Au on $\mathrm{SrTiO}_{3}(001)$ [15], a vastly different system.

Single crystal $\mathrm{NaCl}$ substrates (Korth Kristalle, Germany) were cleaved in UHV with a base pressure of $3 \times 10^{-8} \mathrm{~Pa}$, and heated for $1 \mathrm{~h}$ at $\sim 175^{\circ} \mathrm{C}$, yielding $(001)$ surfaces with flat terraces of up to $1.2 \mu \mathrm{m}$. Submonolayer coverages of PTCDA (Acros Organics 98\% purity) were deposited on the room temperature substrates by thermal evaporation at $300^{\circ} \mathrm{C}$ after outgassing overnight at 200-220 ${ }^{\circ} \mathrm{C}$. Coverages of $0.42 \mathrm{ML}-0.85 \mathrm{ML}$ were deposited at rates of $0.007-0.009 \mathrm{ML} / \mathrm{s}$ as measured by a quartz crystal microbalance.

A commercial JEOL JSPM 4500a UHV AFM operated at room temperature was used to study the molecular films 


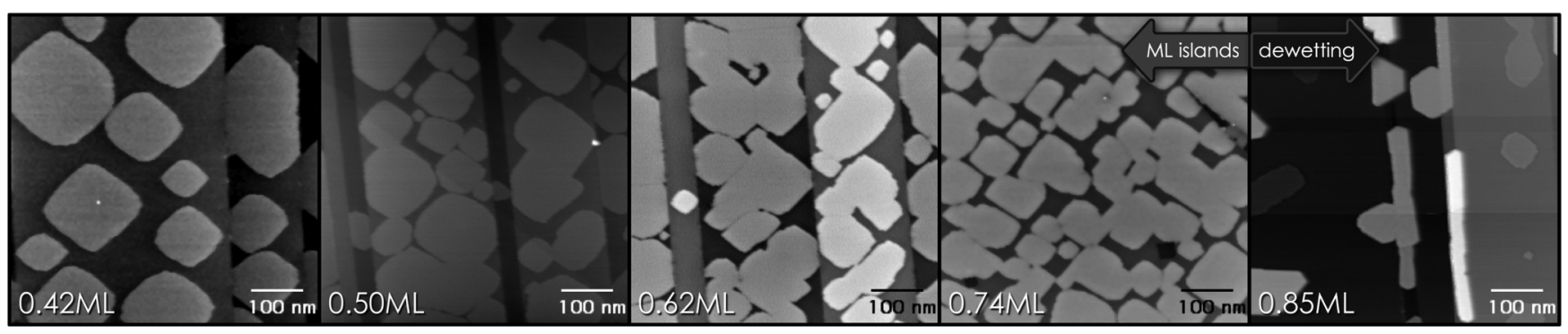

FIG. 1. Overview images $(600 \times 600 \mathrm{~nm})$ of different submonolayer coverages of PTCDA on NaCl. At $0.74 \mathrm{ML}$ and below, only monolayer islands are observed, at $0.85 \mathrm{ML}$, monolayer islands and tall crystalline islands coexist.

by nc-AFM. Excitation of the cantilever and measurement of the frequency shift caused by the tip-sample interaction is performed with a NanoSurf easyPLL which also provides constant amplitude feedback. Nanosensors cantilevers (PPP-NCLR) were used, which have typical resonance frequencies of $170 \mathrm{kHz}$ and spring constants of 40-50 N/m. Typical oscillation amplitudes used were 6$7 \mathrm{~nm}$, and typical $Q$ factors were $\sim 6000$.

Several submonolayer coverages of PTCDA were deposited and characterized by nc-AFM (see Fig. 1). At coverages of $0.74 \mathrm{ML}$ and below, roughly square-shaped monolayer islands with rounded corners showing alignment with $\langle 110\rangle$ directions of the substrate were observed. To confirm that these are indeed monolayer islands, heights were measured with Kelvin probe feedback to compensate for differing electrostatic forces [16] over the PTCDA and $\mathrm{NaCl}$ (supporting information, part 1 [17]). The height of the islands was found to be $3.4 \pm 0.2 \AA$ which corresponds closely to the layer to layer spacing of (102) planes of PTCDA crystals of $3.3 \AA$ [18]. At $0.85 \mathrm{ML}$, coexistence of two different types of islands was observed: the same

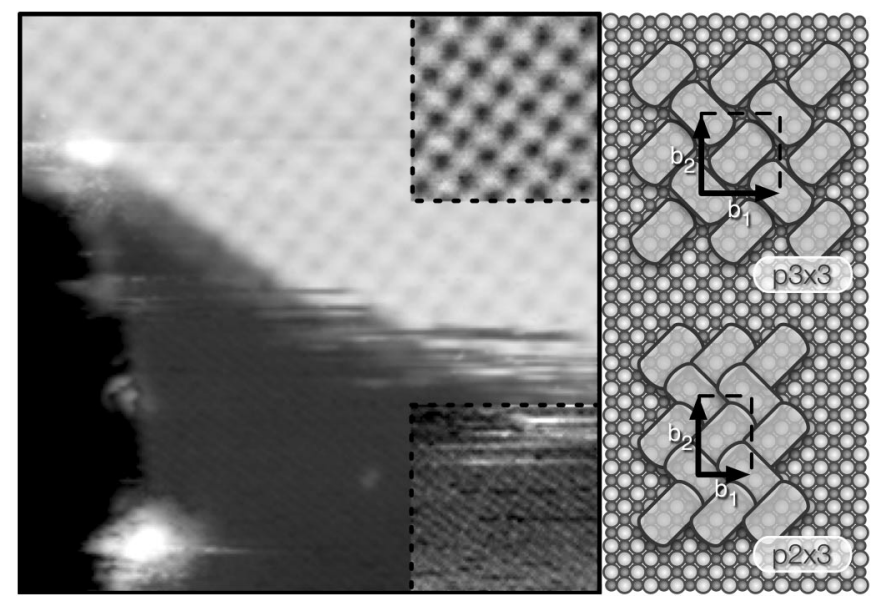

FIG. 2. Left: nc-AFM topography (raw data) of a monolayer island showing molecular and atomic scale resolution on the overlayer and substrate with insets adjusted for contrast $(\Delta f=$ $-8.7 \mathrm{~Hz}$, size: $20 \mathrm{~nm}, z$ scale $=5.5 \AA$ ). Right: schematic of proposed $p 3 \times 3$ monolayer epitaxy (top) and proposed $p 2 \times 3$ multilayer interface structure (bottom). monolayer islands as described for lower coverages, and taller islands showing crystallographic edges typical for PTCDA growth on other surfaces such as $\mathrm{KBr}$ [11].

High resolution imaging over the edge of a PTCDA monolayer island (see Fig. 2) allows determination of the epitaxial relation of the overlayer by calibration of all measured distances to the known lattice of the substrate. The monolayer islands exhibit a $p 3 \times 3$ epitaxy (shown schematically on the upper right of Fig. 2) where the center molecule has the opposite orientation to those at the corners of the unit cell, similar to the molecular arrangement of the square phase observed for PTCDA on other surfaces [18-20]. This $p 3 \times 3$ structure introduces a rather large strain, as compared to a $p 2 \times 3$ structure, for example (see Table I), which was observed for PTCDA on $\mathrm{KBr}$ [12]. While the $p 3 \times 3$ structure clearly forms, and may be allowed in a soft molecular system, it is not readily compatible with the typical herringbone arrangement observed for PTCDA, and one may infer that as multilayer structures form, the molecular arrangement at the surface is altered to accommodate the additional layers.

To investigate the stability of these structures and the effect of the addition of a second layer, molecular mechanics (MM) calculations were carried out using a force field which takes into account both electrostatic and van der Waals interaction, methods described in [21]. Density functional (DFT) calculations were first performed to confirm that there is no significant contribution to the interaction through electronic hybridization or charge transfer between PTCDA and the $\mathrm{NaCl}(001)$ surface (supporting information, part 2 [17]). Both DFT and MM methods were used to determine the Cl-top site as the

TABLE I. Percent change from bulk PTCDA lattice parameters of the (102) planes of the $\alpha\left(b_{1}=1.196 \mathrm{~nm}, b_{2}=\right.$ $1.991 \mathrm{~nm})$ and $\beta\left(b_{1}=1.245 \mathrm{~nm}, b_{2}=1.930 \mathrm{~nm}\right)$ [9] crystal structures for $p 3 \times 3$ and $p 2 \times 3$ epitaxial structures on $\mathrm{NaCl}$ $(a=0.56 \mathrm{~nm})$.

\begin{tabular}{ccccc}
\hline \hline & $p 3 \times 3$ & & $p 2 \times 3$ & \\
& $\Delta b_{1}$ & $\Delta b_{2}$ & $\Delta b_{1}$ & $\Delta b_{2}$ \\
\hline$\alpha$ & $40 \%$ & $-16 \%$ & $-6 \%$ & $-16 \%$ \\
$\beta$ & $35 \%$ & $-13 \%$ & $-10 \%$ & $-13 \%$ \\
\hline \hline
\end{tabular}


stable adsorption site, where the center of the molecule resides over $\mathrm{a} \mathrm{Cl}^{-}$ion. In this position, the more negatively charged oxygen groups at the ends of the molecules reside over the $\mathrm{Na}^{+}$surface ions as a result of electrostatic interactions. Additionally, both the DFT calculations and MM calculations found molecule-substrate distances closely corresponding to the measured height of the monolayer islands: $3.5 \AA$ molecule-substrate distance from DFT, and $2.73 \AA$ closest and $3.71 \AA$ farthest $\mathrm{O}-\mathrm{Na}^{+}$distance from MM while a layer height of $3.4 \pm 0.2 \AA$ was determined from experiments. This good agreement between DFT and MM, as well as the lack of a significant electronic contribution to the binding, allows the use of the MM method which can better model the intermolecular interactions, primarily van der Waals in origin, as well as electrostatic interactions, shown to contribute significantly to the molecule-substrate interaction (supporting information, part 2 [17]).

In the MM calculations, both the $p 3 \times 3$, and proposed $p 2 \times 3$ structures were considered. The $p 2 \times 3$ structure was found to be energetically favorable overall in which the molecules adopt a small tilt away from the usual coplanar adsorption geometry to accommodate the close packing required for this structure (see Fig. 3). However, while the intermolecular interactions are comparable to the substrate-molecule interaction for the $p 2 \times 3$ structure, for the $p 3 \times 3$ structure the molecule-substrate interaction dominates (see Table II). In forming a monolayer the $p 3 \times$ 3 structure maximizes the interaction with the surface, though this structure is metastable.

As the $p 3 \times 3$ structure differs significantly from the bulk PTCDA crystal structures, the addition of a second layer was modeled for both the $p 3 \times 3$ and $p 2 \times$ 3 structures by using a $3 \times 6$ cell. For both interface structures, several initial second-layer configurations were tested; however, for the $p 3 \times 3$ interface no ordered second layer could be found (see Fig. 3). This is consistent with the findings of Gustafsson et al. that upper layers were not observed on the square phase regions [18]. For the $p 2 \times 3$ interface, the second layer forms a highly ordered structure with lattice parameters very close to those of the $\alpha$-PTCDA crystal, differing only by $+5 \%,-2 \%$, and $+0.3 \%$ for $b_{1}, b_{2}$, and interlayer spacing, respectively. This supports the idea that while a $p 3 \times 3$ monolayer can form initially, the structure is incompatible with a multilayer crystal and thus we anticipate that the interface adapts to accommodate the growth of additional layers. This change in the interface structure would also be accompanied by a change in the interface energy and surface energy of the molecular crystal resulting in the observed dewetting transition.

As the $p 3 \times 3$ structure is extended in one direction, the molecular packing is less dense than in the bulk crystal which was used for the definition a ML. A completely covered surface is expected at $0.85 \mathrm{ML}$ (for $\beta$ ) for the
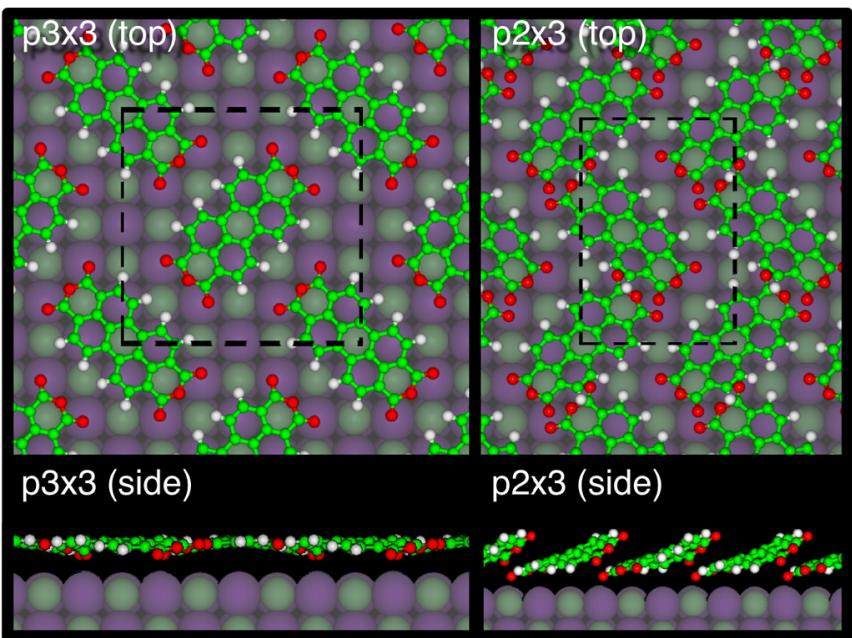

p2×3 (side)

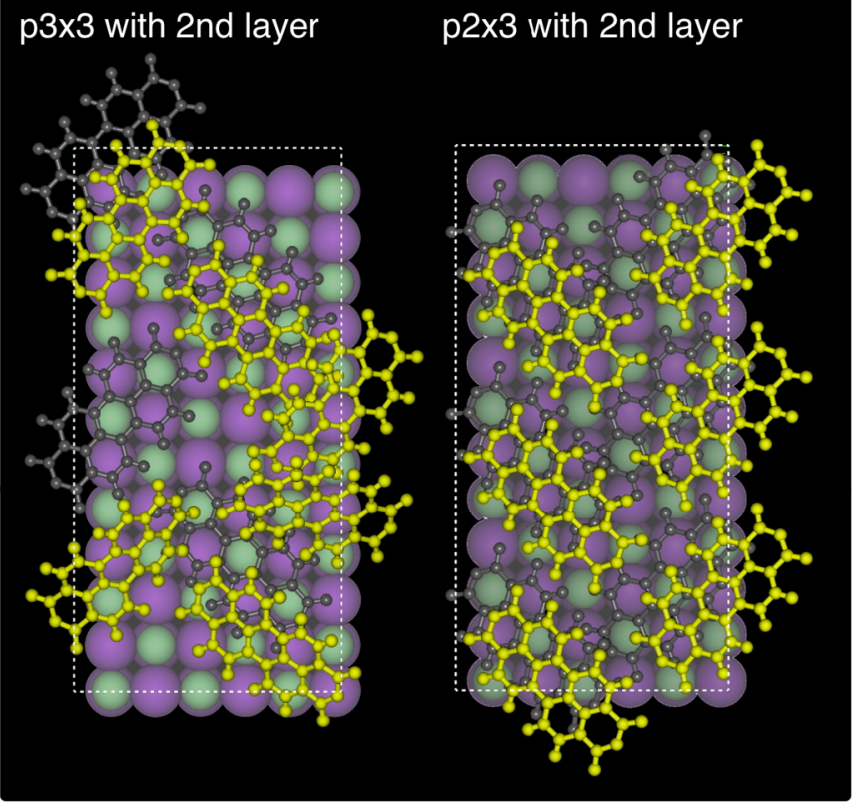

FIG. 3 (color online). Top: Top and side views of $p 3 \times 3$ and $p 2 \times 3$ structures. Bottom: disordered and ordered second-layer (yellow or light gray) on a $p 3 \times 3$ (gray) and a $p 2 \times 3$ interface layer, respectively.

$p 3 \times 3$ structure, indicating that such a dewetting transition should occur near this coverage, as is indeed observed. Additionally, the transition from the less dense monolayer epitaxy to a structure resembling the bulk PTCDA crystal structure is consistent with the spectroscopic observations of Schlettwein et al. [10] exhibiting a shift in fluorescence

TABLE II. Calculated adsorption energies for $p 2 \times 3$ and $p 3 \times 3$ with intermolecular and molecule-substrate contributions.

\begin{tabular}{cccc}
\hline \hline & $E_{\text {adsorption }}$ & $E_{\text {molecule-molecule }}$ & $E_{\text {molecule-substrate }}$ \\
\hline$p 2 \times 3$ & $-30.2 \mathrm{kcal} / \mathrm{mol}$ & $-13.4 \mathrm{kcal} / \mathrm{mol}$ & $-16.8 \mathrm{kcal} / \mathrm{mol}$ \\
$p 3 \times 3$ & $-27.8 \mathrm{kcal} / \mathrm{mol}$ & $-7.0 \mathrm{kcal} / \mathrm{mol}$ & $-20.8 \mathrm{kcal} / \mathrm{mol}$ \\
\hline \hline
\end{tabular}




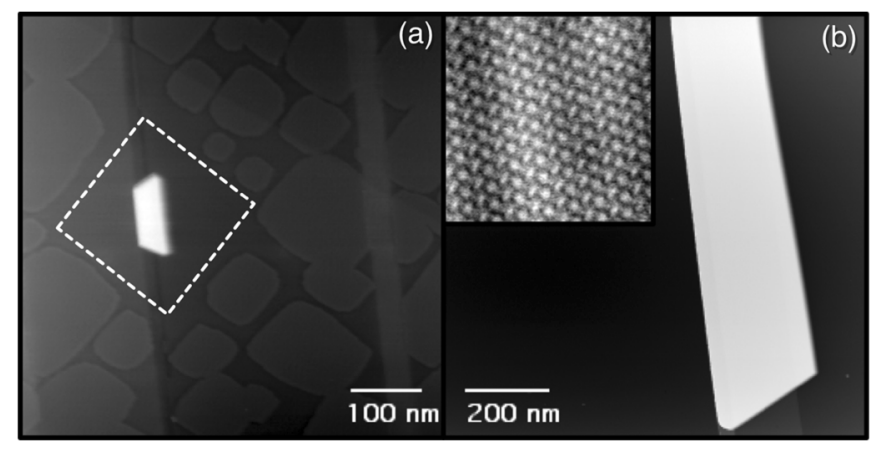

FIG. 4. (a) A tall crystalline island surrounded by a depletion region (indicated by the dashed box) caused by substrate induced dewetting ( $\Delta f=-8.4 \mathrm{~Hz}, z$ scale: $3.6 \mathrm{~nm}$ ). (b) PTCDA island after annealing $(\Delta f=-2.3 \mathrm{~Hz}, z$ scale $=7 \mathrm{~nm})$. Inset: molecular resolution of the herringbone structure observed on top of the island $(\Delta f=-25.5 \mathrm{~Hz}$, size: $15 \mathrm{~nm}, z$ scale $=0.1 \AA)$.

from monomerlike signatures to bulklike excimeric signatures starting near $1 \mathrm{ML}$.

Substrate morphology also appears to induce local dewetting. In regions where the substrate has a high step density or narrow troughs [such as in Fig. 4(a)], multilayer crystalline islands are observed in some instances at coverages below the dewetting transition. On top of these tall crystallites the familiar herringbone arrangement with larger scale striped features and dislocation lines or grain boundaries is observed (not shown), indicative of a bulklike structure under strain. These islands are surrounded by a depletion region, void in monolayer islands of an area closely corresponding to the volume of molecules in the crystallite. For example, the depleted region around the crystalline island in Fig. 4(a) represents a volume of $\sim 16300 \mathrm{~nm}^{3}$ if covered by monolayer islands, while the volume of the crystalline island observed is $15900 \mathrm{~nm}^{3}$. Such a depletion region further indicates that the crystallites are formed by removal of the monolayer islands rather than two types of islands which grow independently.

Annealing of the $0.74 \mathrm{ML}$ coverage, which had predominantly monolayer islands over the majority of the surface, at $150^{\circ}$ for $16 \mathrm{~h}$ resulted in large crystalline islands [shown in Fig. 4(b)] sometimes several microns apart. These islands were found to have heights upwards of $5 \mathrm{~nm}$ corresponding to 15 or more layers, and were often several microns in length. A clear herringbone lattice with a longer range topographic pattern on the top surface was observed [see Fig. 4(b) inset] indicating a strained bulklike structure, similar to that seen on those crystalline islands observed with no annealing. No evidence of the metastable monolayer islands was found after annealing.

In summary, we have presented an example of a dewetting transition in a molecular system which is driven by strain, and is accompanied by a change in crystal structure. It is proposed that the observed monolayer islands are metastable and are incompatible with a multilayer structure. Thus, as coverage is increased beyond the first monolayer the interface structure is adapted to accommodate the growth of additional layers triggering a dewetting transition. This is supported by MM calculations which show that while there is no ordered structure possible on the $p 3 \times 3$ interface, the second layer on the more stable $p 2 \times$ 3 interface is not only ordered, but similar to the bulk crystal structure for PTCDA. The observation of dewetting in this prototype molecule-insulator system and the determination of the molecular scale origin of the dewetting transition is an important step in understanding key growth processes of such systems.

The authors wish to thank NSERC, CIfAR, FQRNT, and CFI for support, as well as the MOST, NSFC, CAS, SCCAS, and SSC for support of the theoretical work.

Note added. - A publication recently appearing in Advanced Materials by Dienel et al. has come to our attention in which PTCDA on $\mathrm{KCl}$ was investigated exhibiting a similar dewettting of a monolayer structure [22].

[1] C. D. Dimitrakopoulos and P. R. L. Malenfant, Adv. Mater. 14, 99 (2002).

[2] G. Horowitz, Adv. Mater. 10, 365 (1998).

[3] G. Witte and C. Woll, J. Mater. Res. 19, 1889 (2004).

[4] N. Karl, Synth. Met. 133, 649 (2003).

[5] G. Witte, K. Hanel, S. Sohnchen, and C. Woll, Appl. Phys. A 82, 447 (2006).

[6] D. Kafer, L. Ruppel, and G. Witte, Phys. Rev. B 75, 085309 (2007).

[7] S. Rath and H. Port, Chem. Phys. Lett. 421, 152 (2006).

[8] S. A. Burke, J. M. Mativetsky, S. Fostner, and P. Grutter, Phys. Rev. B 76, 035419 (2007).

[9] T. Ogawa et al., Acta Crystallogr. Sect. B 55, 123 (1999).

[10] D. Schlettwein et al., Chem. Mater. 10, 601 (1998).

[11] T. Kunstmann et al., Phys. Rev. B 71, 121403(R) (2005).

[12] J. M. Mativetsky, S. A. Burke, S. Fostner, and P. Grutter, Nanotechnology 18, 105303 (2007).

[13] J. M. Mativetsky, S. A. Burke, S. Fostner, and P. Grutter, Small 3, 818 (2007).

[14] S. A. Burke, J. M. Mativetsky, R. Hoffmann, and P. Grutter, Phys. Rev. Lett. 94, 096102 (2005).

[15] F. Silly and M. R. Castell, Phys. Rev. Lett. 96, 086104 (2006).

[16] S. Sadewasser and M. C. Lux-Steiner, Phys. Rev. Lett. 91, 266101 (2003).

[17] See EPAPS Document No. E-PRLTAO-100-071820 for supporting information. For more information on EPAPS, see http://www.aip.org/pubservs/epaps.html.

[18] J. B. Gustafsson, H. M. Zhang, and L.S. O. Johansson, Phys. Rev. B 75, 155414 (2007).

[19] S. Mannsfeld et al., Org. Electron. 2, 121 (2001).

[20] J. C. Swarbrick et al., J. Phys. Chem. 109, 12167 (2005).

[21] D. Shi et al., Phys. Rev. Lett. 96, 226101 (2006).

[22] T. Dienel et al., Adv. Mater. 20, 959 (2008). 